\title{
Tendência de Realização da Citologia Oncótica e Fatores Associados em Mulheres de 25 a 64 anos
}

\author{
Tendency of performing Cytology Smear and Associated Factors \\ in Women 25 to 64 Years Old
}

\section{RESUMO}

Objetivo: Conhecer a tendência temporal de realização da citologia oncótica e identificar fatores associados à realização do exame em algum momento da vida e nos últimos três anos. Metodologia: Trata-se de um estudo epidemiológico, utilizando dados autorreferidos por mulheres, de 25 a 64 anos, oriundos do estudo transversal Vigitel. Para análise da tendência, dados de 2007 a 2015 da Capital João Pessoa, $\mathrm{PB}$, foram analisados por meio de regressão linear simples e, para identificação dos fatores associados, razões de prevalências foram estimadas utilizando-se regressão de Poisson com dados de 2015. Resultados: A realização da citologia oncótica permaneceu, praticamente, estável no período de 2007 a 2015. Dentre as variáveis analisadas, a faixa etária manteve-se associada a uma maior prevalência de realização do exame nos dois desfechos e o estado civil casado/união estável manteve-se associado à maior prevalência de realização do exame em algum momento da vida; a escolaridade de 9 a 11 anos de estudo apresentou uma associação negativa com a realização do exame nos últimos três anos. Conclusão: A cobertura da citologia oncótica não foi satisfatória entre as mulheres de João Pessoa, Paraíba. A faixa etária mais avançada e as mulheres casadas ou em união estável apresentaram maior prevalência de realização do exame.

\section{DESCRITORES}

Neoplasias. Neoplasias do Colo do Útero. Teste de Papanicolaou. Estudos Transversais. Epidemiologia.

\begin{abstract}
Objective: Know the time trend of performing cytology smears and identifying factors associated with performing the examination at some point in life and in the last three years. Methodology: It is an epidemiological study using self-reported data by women from 25 to 64 years old, from the crosssectional Vigitel study. For the trend analysis, data from 2007 to 2015 in the city of João Pessoa, Paraiba, were analyzed by means of simple linear regression, and to identify associated factors, prevalence ratios were estimated using Poisson Regression, with data from 2015. Results: Oncotic cytology was practically stable from 2007 to 2015 . Among the analyzed variables, age remained associated with a higher prevalence of performing the examination in both outcomes and the marital status married/in a stable union remained associated with a higher prevalence of performing the examination at any lifetime; education from 9 to 11 years of schooling showed a negative association with the completion of the exam in the last three years. Conclusion: The coverage of the Pap smear exam was not satisfactory among women in Joao Pessoa. The advanced age and married/in a stable union woman had higher prevalence of performing the examination.
\end{abstract}

\section{DESCRIPTORS}

Neoplasias. Cervix Neoplasias. Papanicolaou Test. Crosssectional Studies. Epidemiology.

\footnotetext{
${ }^{1}$ Mestra em Ciências da Nutrição. Nutricionista no Restaurante Universitário da Universidade Federal da Paraíba (UFPB) - CAMPUS III, Bananeiras (PB), Brasil.

${ }^{2}$ Doutora. Professora Associada do Departamento de Nutrição, Universidade Federal da Paraíba (UFPB), João Pessoa (PB), Brasil.
} 


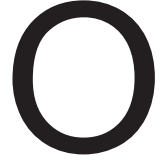
câncer de colo uterino é um importante problema de saúde pública no Brasil e em outros países em desenvolvimento ${ }^{1}$. É a segunda neoplasia maligna mais comum entre as mulheres no mundo, sendo responsável por cerca de 471 mil novos casos e por cerca de 230 mil óbitos de mulheres por ano².

É o segundo tumor mais diagnosticado em mulheres no Brasil, apesar de possuir alto potencial de prevenção e cura quando diagnosticado precocemente ${ }^{2,3}$. Em 2016, a estimativa do câncer de colo uterino, no Brasil, foi de 16.340 novos casos, com um risco estimado de 15,85 casos a cada 100.000 mulheres ${ }^{4}$. Excluídos os tumores de pele não melanoma, o câncer cervicouterino é o mais incidente na Região Norte, o segundo mais incidente nas Regiões Centro-Oeste e Nordeste e corresponde ao terceiro câncer com maior incidência nas Regiões Sul e Sudeste ${ }^{1}$.

Na região Nordeste do Brasil e em João Pessoa, Capital da Paraíba, considerando os grandes grupos de causas de óbitos nas mulheres, dados de 2011 informados pelo Departamento de Informática do Sistema Único de Saúde (Datasus) ${ }^{5}$, mostraram que as neoplasias corresponderam, respectivamente, a uma proporção de óbitos de 16,16\% e $19,48 \%$ quando comparados ao total de óbitos por todas as causas.

O método de rastreamento preconizado para a detecção precoce da doença no Brasil é através da realização do exame citopatológico ou citologia oncótica, conhecido popularmente como exame de Papanicolau; e o Ministério da Saúde preconiza como grupo prioritário para realização deste exame mulheres entre 25 a 64 anos de idade que já tiveram iniciado a atividade sexual6-8. Recomenda-se a sua realização uma vez por ano e, após dois exames anuais consecutivos negativos, a cada três anos ${ }^{1}$. A organização Mundial de Saúde (OMS) destaca que a incidência desse câncer aumenta nas mulheres com idade entre 30 e 39 anos, atingindo seu pico na quinta ou sexta décadas de vida. Os exames antes dos 25 anos não se justificam por predominarem as infecções por papilomavírus humano (HPV), que tendem a regredir espontaneamente e, após os 65 anos de idade, o risco de desenvolvimento do câncer cervical é reduzido dada á sua lenta evolução. Essas evidências justificam a faixa etária de 25 a 64 anos de escolha para o rastreamento ${ }^{7}$.

Embora o Brasil tenha sido um dos primeiros países no mundo a realizar a citologia oncótica, dados mostram que os índices de mortalidade por câncer cervical não registraram nenhuma queda nos últimos vinte anos ${ }^{6}$.

A citologia oncótica consiste no esfregaço de células oriundas da ectocérvice e da endocérvice, que são extraídas por raspagem do colo do útero. Embora ele represente o instrumento mais adequado, prático e de baixo custo para o rastreamento do câncer de colo de útero, devido à forte influência na redução da morbi-mortalidade por este tipo de câncer, a adesão ao exame ainda está distante da cobertura preconizada pelo Ministério da Saúde, que é de 80 a $85 \%{ }^{2}$.

No Brasil, segundo estimativas 
nacionais, cerca de seis milhões de mulheres entre 35 a 49 anos nunca realizaram a citologia oncótica. Considerando que nesta faixa etária ocorre maior número de casos positivos de câncer do colo do útero, tal situação concorre para o diagnóstico do câncer cervicouterino em estágios avançados, com menor sobrevida média quando comparada a dos países desenvolvidos ${ }^{2}$.

A partir de 2007, o Sistema de Vigilância de Fatores de Risco e Proteção para Doenças Crônicas Não Transmissíveis por Inquérito Telefônico (Vigitel), inseriu, em seu questionário eletrônico, perguntas sobre exames preventivos de câncer de colo de útero, tornando possível o monitoramento das coberturas autorreferidas tanto nas capitais brasileiras quanto no Distrito Federal ${ }^{7}$.

Considerando que o acesso ao exame citopatológico é a condição necessária para a eficácia do programa de rastreamento do câncer de colo uterino, a presente pesquisa tem como objetivo: descrever a tendência temporal de autorrelato de realização da citologia oncótica entre os anos de 2007 a 2015 e identificar os fatores associados à realizaçãodo exame, tanto em algum momento da vida quanto nos últimos três anos, referidos por mulheres na faixa etária de 25 a 64 anos, residentes em uma capital do Nordeste do Brasil.

\section{METODOLOGIA}

Realizou-seumestudoepidemiológico, de base populacional, que utilizou dados do Vigitel, um estudo transversal que consiste de um sistema de monitoramento implantado pelo Ministério da Saúde em 2006, cujo objetivo principal é o monitoramento contínuo da frequência e distribuição de fatores de risco e proteção para doenças crônicas não transmissíveis na população brasileira $^{9}$. O projeto de implantação do Vigitel foi aprovado pelo Comitê Nacional de Ética em Pesquisa para Seres Humanos (CONEP) do Conselho Nacional de Saúde (CNS), Ministério da Saúde, sob o Parecer $n^{\circ}$ 355.590, de 26 de Junho de 2013 e Certificado de Apresentação para Apreciação Ética - CAAE n 16202813.2.0000.0008. Este estudo apresenta dois recortes: uma análise de tendência temporal e uma investigação de fatores associados à realização da citologia oncótica, ambos utilizando dados de mulheres entrevistadas com idade de 25 a 64 anos, residentes em domicílios servidos por pelo menos uma linha telefônica fixa, na Capital João Pessoa, Paraíba, Brasil. Para a descrição da tendência temporal de realização da citologia oncótica em algum momento da vida e nos últimos três anos anteriores à entrevista, foram analisadas informações do inquérito Vigitel, realizado entre os anos de 2007 a 2015; e, para análise de associação entre as variáveis independentes e os desfechos, utilizou-se a base de dados do Vigitel 2015.

No ano de 2015, o Vigitel entrevistou 736 mulheres com idade de 25 a 64 anos, público alvo do presente estudo. Esta amostra de 736 mulheres foi ponderada por gênero, idade e escolaridade, considerando metodologia adotada pelo Vigitel, usando 
o método rake ${ }^{9-12}$, tornando os dados desta amostra representativos da população adulta total desta capital. Detalhes do processo de amostragem, ponderação das estimativas do Vigitel, questionário eletrônico utilizado no momento das entrevistas, bem como outros detalhes da metodologia utilizada pelo sistema podem ser vistos em outras publicações ${ }^{9,11}$.

As variáveis dependentes analisadas neste estudo foram: prevalência da realização da citologia oncótica em algum momento da vida e a prevalência da realização do exame nos últimos três anos. As informações sobre a realização do exame foram obtidas por meio das seguintes perguntas, respectivamente: 'A sra. já fez, alguma vez, exame de Papanicolau, exame preventivo de câncer de colo do útero?.' 'E "Quanto tempo faz que a sra. fez exame de Papanicolau?'.

As variáveis independentes analisadas incluem como características demográficas, a faixa etária (25-34; 35-44; 45-54; 55-64 anos); o estado civil (solteira/separada/ divorciada; casada/união estável; viúva) e a escolaridade (0-8; 9-11 e $\geq 12$ anos de estudo). Como indicador de saúde, utilizouse a autoavaliação de saúde, a partir da resposta dada à questão 'A sra classificaria seu estado de saúde como: muito bom, bom, regular, ruim ou muito ruim?. Essa variável foi dicotomizada em sim ou não, considerando mulheres que autoavaliaram sua saúde como ruim ou muito ruim. $\mathrm{O}$ indicador de uso dos serviços de saúde foi a posse de plano de saúde (sim ou não).
Todas as análises estatísticas foram realizadas utilizando o procedimento Stata Survey versão $11 \mathrm{SE}$, usando os fatores de ponderação atribuídos a cada indivíduo entrevistado nos inquéritos do Vigitel. A análise de tendência da série temporal para os dois indicadores foi estratificada segundo a escolaridade das mulheres. A técnica utilizada para estimar a tendência foi o modelo de regressão linear simples, cuja variável resposta (Yi) é a proporção do indicador e a variável explicativa (Xi) no tempo (ano do levantamento). O sinal negativo do coeficiente angular $(\beta)$ da reta ajustada pelo modelo indica que a relação entre o indicador e o tempo é decrescente, caso contrário, a relação é crescente. O valor do coeficiente angular positivo representa o aumento médio anual na proporção do indicador para cada unidade de tempo, caso contrário representa a queda média anual da proporção.

Para a análise dos fatores associados, foram realizados testes de associação pelo qui-quadrado de Pearson para verificar a existência de associação estatística entre as variáveis independentes e os desfechos (valor de $p \leq 0,05$ ). Posteriormente, utilizouse o modelo de regressão de Poisson para verificar a existência de fatores associados aos desfechos. Foram consideradas para introdução no modelo multivariado as variáveis que apresentaram $p \leq 0,20$ na análise univariada. As magnitudes das associações encontradas foram medidas pelas razões de prevalência (RP) com seus respectivos intervalos de confiança (IC95\%). 


\section{RESULTADOS}

A partir da análise de tendência temporal, noperíodo de2007a2015, observouse uma estabilidade na cobertura dos exames de citologia oncótica, apresentando uma prevalência com patamar abaixo de $80 \%$ na realização do exame tanto em algum momento da vida quanto nos últios três anos. Mesmo com a estabilidade, persistem diferenças, segundo a escolaridade, tanto na realização do exame em algum momento da vida quanto nos últimos três anos: em 2015, mulheres com 0-8 anos de estudo tiveram cobertura do exame em algum momento da vida de $84,2 \%$, com 9 a 11 anos de $76,3 \%$ e com 12 anos e mais, $78,8 \%$ e, nos últimos três anos, mulheres com 0-8 anos de estudo apresentaram cobertura do exame de $75,6 \%$, com 9 a 11 anos de $68,4 \%$ e com 12 anos e mais, $75 \%$ (Tabela 1 ).

No recorte transversal, referente especificamente ao ano de 2015, observouse o perfil da população estudada e os fatores associados aos desfechos. Do total de mulheres entrevistadas, a maioria tinha idade entre 45-54 anos, eram casadas, referiram possuir 12 ou mais anos de estudo, não consideraram seu estado de saúde como ruim e referiram não possuir plano privado de saúde (Tabela 2).

As Tabelas 3 e 4 descrevem a análise bruta e ajustada de fatores associados à realização da citologia oncótica em algum momento da vida e à realização do exame nos últimos três anos, no último ano da série, em 2015, respectivamente. Para a realização da citologia oncótica em algum momento da vida, a análise ajustada mostrou

Tabela 1. Variações no percentual de mulheres (25 a 64 anos) que realizaram citologia oncótica em algum momento de suas vidas e nos últimos três anos no período de 2007 à 2015, segundo escolaridade. João Pessoa/PB - Brasil (Vigitel, 2007-2015)

\begin{tabular}{|c|c|c|c|c|c|c|c|c|c|c|c|c|}
\hline & 2007 & 2008 & 2009 & 2010 & 2011 & 2012 & 2013 & 2014 & 2015 & $\mathrm{p}$ valor* & $\begin{array}{l}\text { Inclina- } \\
\text { ção }\end{array}$ & $\begin{array}{l}\text { Inter- } \\
\text { cepto }\end{array}$ \\
\hline $\begin{array}{l}\text { Citologia } \\
\text { oncótica em } \\
\text { algum } \\
\text { momento da } \\
\text { vida }\end{array}$ & 79,7 & 83,6 & 78,3 & 80,6 & 80,3 & 83,8 & 76,8 & 77,2 & 78,7 & 0,3552 & $-0,317$ & 716,8 \\
\hline \multicolumn{13}{|l|}{ Escolaridade } \\
\hline $0-8$ anos & 79,6 & 80,8 & 79,9 & 79 & 79,3 & 85,6 & 79,7 & 78 & 84,2 & 0,4456 & 0,27 & $-462,3$ \\
\hline 9-11 anos & 78,2 & 84,6 & 73,9 & 80,7 & 77,4 & 79,3 & 74,4 & 78,5 & 76,3 & 0,3315 & $-0,438$ & 959,6 \\
\hline 12 ou mais & 82,1 & 88,2 & 81,9 & 83,3 & 85,9 & 87,9 & 76,5 & 74,3 & 78,8 & 0,1072 & $-1,018$ & 2130 \\
\hline $\begin{array}{l}\text { Citologia } \\
\text { oncótica nos } \\
\text { últimos três } \\
\text { anos }\end{array}$ & 74,8 & 76,6 & 72,8 & 74 & 72,9 & 77,6 & 70,3 & 72,3 & 72,6 & 0,2068 & $-0,385$ & 848 \\
\hline \multicolumn{13}{|l|}{ Escolaridade } \\
\hline $0-8$ anos & 73,6 & 74,5 & 73,7 & 70,7 & 69,4 & 78,6 & 68,2 & 71,1 & 75,6 & 0,8502 & $-0,088$ & 250,5 \\
\hline 9-11 anos & 74,3 & 75,2 & 69,3 & 74,7 & 70,9 & 72,4 & 71,4 & 74,8 & 68,4 & 0,2698 & $-0,382$ & 839,9 \\
\hline 12 ou mais & 78,1 & 82,3 & 76,3 & 78,8 & 80,9 & 83,8 & 71,5 & 70,7 & 75 & 0,1526 & $-0,863$ & 1813,7 \\
\hline
\end{tabular}


que permaneceram independentemente associada a uma maior prevalência de realização do exame as faixas etárias de 35-44 anos, de 45-54 anos e de 55-64 anos; e o fato da mulher referir ser casada ou estar em união estável (Tabela 3). Para a realização da citologia oncótica nos últimos três anos, a análise ajustada mostrou que houve associação a uma maior prevalência de realização do exame, as faixas etárias de 35-44 anos, de 45-54 anos e de 55-64 anos, já a escolaridade na faixa de 9-11 anos de estudo apresentou, na análise ajustada, uma associação inversa com a realização do exame nos últimos três anos (Tabela 4).

\section{DISCUSSÃO}

O estudo atual analisa dados do Vigitel, referentes à Cidade de João Pessoa, Paraiba e indica que, entre os anos de 2007 - 2015, as prevalências do relato de realização da citologia oncótica, tanto em algum momento da vida quanto nos últimos três anos anteriores, permaceram estáveis e, no geral, com percentual de realização

Tabela 2. Distribuição percentual das mulheres entrevistadas segundo variáveis sóciodemográficas, indicador de saúde e indicador de uso dos serviços de saúde. João Pessoa/PB - Brasil (2015)

\begin{tabular}{l|l|l|c}
\hline Variáveis & $\mathrm{P}(\%)^{*}$ & IC95\%** & Valor de $\mathrm{p}^{* * *}$ \\
\hline Sóciodemográficas & & & \\
Faixa etária & 21,6 & $18,0-25,7$ & 0,163 \\
25-34 anos & 23,6 & $20,1-27,5$ & \\
35-44 anos & 28,8 & $24,8-33,1$ & \\
45-54 anos & 26,0 & $22,0-30,4$ & \\
55-64 anos & 42,1 & $37,6-46,8$ & 0,000 \\
Estado civil & 53,9 & $49,2-58,5$ & \\
Solteiro/separado/divorciado & 4,0 & $2,5-6,2$ & 0,000 \\
Casado/união estável & & & \\
Viúvo & 20,3 & $17,0-24,1$ & \\
Escolaridade & 35,7 & $31,5-40,0$ & 0,000 \\
0-8 anos & 44,1 & $39,4-48,8$ & \\
9-11 anos & & & \\
$\geq 12$ anos & & & \\
Indicador de saúde & 95,2 & $93,0-96,8$ & \\
Autoavaliação de saúde como ruim & 4,8 & $3,2-7,0$ & \\
Não & & & \\
Sim & 50,2 & $45,6-54,8$ & \\
Indicador de uso de serviços de saúde & 49,8 & $45,2-54,4$ & \\
Posse de plano de saúde & & \\
Não & &
\end{tabular}

Nota: os resultados expressam o percentual da população.

* Prevalência.

** Intervalo de confiança

${ }^{* * \star}$ Análise estatística realizada: teste Qui-quadrado de Pearson. 
Tabela 3. Razões de prevalência (bruta e ajustada) para autorrelato da realização de citologia oncótica em algum momento da vida, de acordo com variáveis sóciodemográficas, indicador de saúde e indicador de uso dos serviços de saúde. João Pessoa/PB - Brasil (2015)

\begin{tabular}{|c|c|c|c|c|}
\hline \multirow[b]{2}{*}{ Variáveis } & \multicolumn{4}{|c|}{ Realização de Citologia oncótica } \\
\hline & $\begin{array}{l}\text { RP }^{*} \text { Bruta } \\
\text { IC95\%** }\end{array}$ & $\mathrm{p}$ & $\begin{array}{l}\mathrm{RP}^{*} \text { Ajustada** }_{\text {IC }}{ }^{*} \%^{*}\end{array}$ & $p$ \\
\hline \multicolumn{5}{|l|}{ Sóciodemográficas } \\
\hline \multicolumn{5}{|l|}{ Faixa Etária } \\
\hline 25-34 anos & 1,0 & & 1,0 & \\
\hline 35-44 anos & $1,27(1,08-1,49)$ & 0,003 & $1,28(1,10-1,50)$ & 0,001 \\
\hline 45-54 anos & $1,34(1,15-1,56)$ & 0,000 & $1,30(1,12-1,50)$ & 0,001 \\
\hline 55-64 anos & $1,38(1,17-1,62)$ & 0,000 & $1,31(1,11-1,54)$ & 0,001 \\
\hline \multicolumn{5}{|l|}{ Estado civil } \\
\hline Solteiro/separado/divorciado & 1,0 & & 1,0 & \\
\hline Casado/união estável & $1,12(1,02-1,23)$ & 0,014 & $1,10(1,01-1,20)$ & 0,027 \\
\hline Viúvo & $1,21(1,04-1,40)$ & 0,010 & $1,11(0,96-1,30)$ & 0,146 \\
\hline \multicolumn{5}{|l|}{ Escolaridade } \\
\hline $0-8$ anos & 1,0 & & 1,0 & \\
\hline 9-11 anos & $0,88(0,78-0,99)$ & 0,036 & $0,91(0,81-1,02)$ & 0,127 \\
\hline$\geq 12$ anos & $1,00(0,91-1,11)$ & 0,872 & $0,98(0,87-1,10)$ & 0,783 \\
\hline \multicolumn{5}{|c|}{ Indicador de saúde } \\
\hline Autoavaliação da saúde com o ruim & 1,0 & & 1,0 & \\
\hline Sim & $0,72(0,51-1,03)$ & 0,076 & $0,72(0,50-1,03)$ & 0,077 \\
\hline \multicolumn{5}{|c|}{$\begin{array}{l}\text { Indicador de uso dos serviços de saúde } \\
\text { Posse de plano de saúde }\end{array}$} \\
\hline $\begin{array}{l}\text { Não } \\
\text { Sim }\end{array}$ & $\begin{array}{c}1,0 \\
1.09(1.00-1.19)\end{array}$ & 0.033 & $\begin{array}{c}1,0 \\
1.08(0,97-1,19)\end{array}$ & 0.133 \\
\hline
\end{tabular}

${ }^{*}$ RP, Razão de Prevalência; IC, Intervalo de Confiança.

${ }^{*}$ Análise ajustada por Regressão de Poisson, realizada apenas com as variáveis independentes que apresentaram significância $(p \leq 0,20)$ no teste Qui-quadrado de Pearson.

do exame abaixo de $80 \%$. Segundo a OMS, com uma cobertura de exame citopatológico do colo do útero da ordem de $80 \%$ na população-alvo do rastreamento e a garantia de diagnóstico e tratamento adequados dos casos alterados, é possível reduzir, em média, de 60 a $90 \%$ a incidência do câncer cervical invasivo ${ }^{13}$.

No presente estudo, os dados do Vigitel 2015, mostraram que a cobertura da citologia oncótica, realizada tanto em algum momento da vida quanto nos últimos três anos, autorrelatada por mulheres de 2564 anos em João Pessoa, foi menor que a cobertura preconizada pelo Ministério da Saúde, que é de 80 a 85\%². Dados do Vigitel ${ }^{11}$, coletados no mesmo ano, mostraram que as maiores frequências de mulheres com idade de 25 a 64 anos que referiram ter realizado citologia oncótica nos últimos três anos foram observadas em Porto Alegre (88,6\%), São Paulo (87,8\%), Curitiba e Vitoria (87,5\%); e, as menores, em Maceió $(65,3 \%)$, Teresina $(70,2 \%)$ e Fortaleza $(72,4 \%)$. 
Tabela 4. Razões de prevalência (bruta e ajustada) de autorrelato da realização de citologia oncótica nos últimos três anos, de acordo com variáveis sociodemográficas, indicador de saúde e indicador de uso dos serviços de saúde. João Pessoa/PB - Brasil (2015)

\begin{tabular}{|c|c|c|c|c|}
\hline \multirow[b]{2}{*}{ Variáveis } & \multicolumn{4}{|c|}{ Realização de Citologia oncótica nos últimos três anos } \\
\hline & $\begin{array}{l}\mathrm{RP}^{*} \text { Bruta } \\
\text { IC95\%* }\end{array}$ & $\mathrm{p}$ & $\begin{array}{l}\text { RP }^{*} \text { Ajustada }^{* *} \\
\text { IC } 95 \% \%^{*}\end{array}$ & $\mathrm{p}$ \\
\hline \multicolumn{5}{|l|}{ Sóciodemográficas } \\
\hline \multicolumn{5}{|l|}{ Faixa etária } \\
\hline $25-34$ anos & 1,0 & & 1,0 & \\
\hline 35-44 anos & $1,19(1,00-1,43)$ & 0,050 & $1,20(1,01-1,43)$ & 0,033 \\
\hline 45-54 anos & $1,29(1,10-1,53)$ & 0,002 & $1,24(1,06-1,46)$ & 0,007 \\
\hline 55-64 anos & $1,33(1,10-1,61)$ & 0,003 & $1,25(1,04-1,51)$ & 0,015 \\
\hline \multicolumn{5}{|l|}{ Estado civil } \\
\hline Solteiro/separado/divorciado & 1,0 & & - & \\
\hline Casado/união estável & $1,15(1,03-1,29)$ & 0,013 & - & - \\
\hline Viúvo & $1,21(0,94-1,55)$ & 0,129 & - & - \\
\hline \multicolumn{5}{|l|}{ Escolaridade } \\
\hline $0-8$ anos & 1,0 & & 1,0 & \\
\hline 9-11 anos & $0,82(0,71-0,95)$ & 0,011 & $0,83(0,72-0,96)$ & 0,016 \\
\hline$\geq 12$ anos & $1,03(0,91-1,16)$ & 0,586 & $0,99(0,86-1,13)$ & 0,906 \\
\hline \multicolumn{5}{|c|}{$\begin{array}{l}\text { Indicador de saúde } \\
\text { Autoavaliação da saúde como ruim }\end{array}$} \\
\hline Não & 1,0 & & 1,0 & \\
\hline Sim & $0,66(0,43-1,00)$ & 0,054 & $0,66(0,43-1,00)$ & 0,059 \\
\hline \multicolumn{5}{|c|}{$\begin{array}{l}\text { Indicador de uso dos serviços de saúde } \\
\text { Posse de plano de saúde }\end{array}$} \\
\hline $\begin{array}{l}\text { Não } \\
\text { Sim }\end{array}$ & $\begin{array}{c}1,0 \\
1.12(1.00-1,24)\end{array}$ & 0.035 & $\begin{array}{c}1,0 \\
1.06(0.94-1,19)\end{array}$ & 0.319 \\
\hline
\end{tabular}

${ }^{*}$ RP, Razão de Prevalência; IC, Intervalo de Confiança.

${ }^{* *}$ Análise ajustada por Regressão de Poisson, realizada apenas com as variáveis independentes que apresentaram significância $(p \leq 0,20)$ no teste Qui-quadrado de Pearson.

A literatura é enfática em sinalizar que a desinformação, o conhecimento errôneo ou insuficiente constituem barreiras à realização de medidas preventivas para o câncer de colo uterino, como a realização da citologia oncótica e, apesar do câncer de colo uterino apresentar altos potenciais de prevenção por meio do rastreio oportunístico, ainda existem mulheres que desenvolvem e morrem por este tipo de câncer no Brasil, pelo fato de desconhecerem a finalidade deste exame, além disso, a inserção da mulher no mercado de trabalho, ao mesmo tempo em que representa conquista em termos sociais, também constitui fator interveniente para a não realização do exame, contribuindo para as mulheres se tornarem mais vulneráveis ao câncer cervicouterino e, deste modo, impedindo o estabelecimento de ações eficazes no âmbito da prevenção². Além dessas hipóteses, são considerados também outros fatores associados à resistência de mulheres ao exame preventivo: desmotivação, receio da dor, vergonha, 
distância, dificuldades para deixar filhos ou parentes, dificuldades financeiras e com transportes para o deslocamento e a não permissão do parceiro para que a mulher realize o exame ${ }^{1,3}$.

Os resultados do presente estudo também identificaram os fatores associados ao autorrelato de realização da citologia oncótica tanto em algum momento da vida quanto nos últimos três anos. Para a realização da citologia oncótica em algum momento da vida, dentre as variáveis investigadas, manteve-se, independentemente, associado a uma maior prevalência deste exame, as faixas etárias de 35-44; 45-54 e 55-64 anos e o fato da mulher referir ser casada ou estar em união estável. Para a realização da citologia oncótica nos últimos três anos, mantiveram-se, independentemente, associados a uma maior prevalência deste exame as faixas etárias de 35-44; 45-54 e 55-64 anos; a escolaridade entre 9-11 anos mostrou uma associação negativa, apresentando menor prevalência de realização deste exame nos últimos três anos que antecederam a entrevista.

As faixas etárias mais avançadas mostraram-se, neste estudo, associadas aos dois desfechos, apresentando uma maior prevalência de realização do exame quando comparadas às mulheres com menor faixa etária. Resultado inverso foi obervado em estudo realizado em cidade do nordeste paulista $^{3}$, onde houve tendência decrescente na cobertura do exame entre as faixas etárias mais avançadas. Vale et al ${ }^{14}$ ressaltam que a maior frequência de eventos em mulheres mais jovens, como gravidez, uso de métodos contraceptivos e tratamentos de leucorréias, influencia uma maior busca por consultas ginecológicas quando comparadas com as de maior idade. Assim, a cobertura, além de insuficiente, concentra-se nas mulheres mais jovens, não alcançando mulheres de 50 a 60 anos, grupo com maior risco para o câncer.

Ser casada ou estar em um relacionamento estável também se manteve associado a uma maior prevalência de realização da citologia oncótica em algum momento da vida quando comparada às mulheres solteiras, separadas ou divorciadas. Resultado semelhante também foi observado em estudo realizado no município de Rio Grande, no sul do Brasil, que detectou que mulheres sem companheiro apresentam maiores prevalências em relação à não realização do exame preventivo do câncer do colo do útero ${ }^{15}$.

A escolaridade é considerada como uma dimensão da condição social, com grau de influência importante na realização de exames preventivos e, no presente estudo, a faixa de escolaridade de 9-11 anos de estudo mostrou-se associada a uma menor prevalência de realização da citologia oncótica nos últimos três anos, quando comparada à menor faixa. Resultado semelhante foi encontrado em estudo realizado no Estado do Maranhão, Nordeste do Brasil ${ }^{16}$, onde a maioria das mulheres que realizaram os exmes citopatológicos possuía baixo nível de escolaridade. Thuler et $\mathrm{al}^{17}$, em estudo observacional dos casos de câncer do colo 
do útero no Brasil, identificaram o ensino fundamental incompleto como a escolaridade mais prevalente, sugerindo ser um fator associado para o desenvolvimento do câncer cervical. Provavelmente esta associação é uma realidade sociodemográfica brasileira, em especial das mulheres que buscam atendimento no Sistema Único de Saúde (SUS). Porém, estudo realizado em Porto Velho, Estado de Rondônia ${ }^{1}$ mostrou que o grau de instrução das mulheres não interferiu na realização do exame preventivo do câncer do colo uterino, embora dados da literatura relatem que a realização do exame está positivamente relacionada ao maior nível de instrução ${ }^{18}$.

Algumas limitações deste estudo devem ser apontadas. Uma limitação a ser destacada refere-se especificamente ao aspecto metodológico adotado pelo sistema Vigitel, o qual entrevista apenas indivíduos residentes em domicílios que possuem telefone fixo, caracterizando um possível viés de aferição, porém, fatores de ponderação são utilizados para estimar as prevalências, visando igualar a mesma distribuição populacional do Vigitel e do Censo. Outra limitação refere-se à realização da citologia oncótica, a qual foi obtida por meio de dados autorreferidos, não podendo se descartar um potencial viés de informação, entretanto, estudos de validação de alguns indicadores do Vigitel foram realizados no Brasil ${ }^{19,20} \mathrm{e}$ mostraramconcordânciaentreasinformações referidas no Vigitel $e$ as informações relatadas em pesquisas domiciliares, com a vantagem de ser um método não invasivo, de fácil obtenção dos dados e baixo custo. Finalmente, por ser um estudo com desenho metodológico transversal, não é possível estabelecer relação de causa e efeito sobre as associações encontradas entre os desfechos e as variáveis independentes.

Apesar das limitações identificadas, algumas potencialidades deste estudo precisam ser destacadas. Estudos transversais de base populacional, com amostras representativas, conduzidas através de pesquisas telefônicas, são de grande relevância, por serem alternativas rápidas e de baixo custo, constituindo uma importante ferramenta epidemiológica para dimensionar os problemas, estimar indicadores de condição de saúde, de comportamentos relacionados à saúde, de acesso e uso dos serviços de saúde e de morbidade referida, criando subsídios para adoção de ações de promoção à saúde e prevenção de doenças na população de referência ou em outras com características semelhantes.

\section{CONCLUSÃO}

Considerando-se a meta preconizada pela OMS de $80,0 \%$ de cobertura do exame de citologia oncótica nos últimos três anos para se conseguir a efetividade do programa de prevenção do câncer de colo uterino, podese afirmar que em João Pessoa, Paraíba, a cobertura está inferior ao preconizado. Além disso, os achados deste estudo identificaram a existência de significativas desigualdades 
socioeconômicas quanto à realização deste exame, reforçando a necessidade de intervenções que visem à promoção da equidade, pois não basta apenas garantir o acesso ao exame preventivo nos serviços de saúde, tão pouco emitir informações acerca do mesmo, antes, é necessário garantir que a mulher tenha acesso a essas informações, e que estas sejam adequadas a sua realidade histórica, social e de saúde, a fim de que sejam compreensíveis e factíveis. Dessa forma, acredita-se que as mulheres resistentes ao exame serão levadas a refletirem acerca dos seus saberes e se conscientizarão da verdadeira importância do mesmo, para que assim, possam efetivamente realizá-lo.

\section{REFERÊNCIAS}

1. Lucena LT, Zãn DG, Crispim PTB, Ferrari JO. Fatores que influenciam a realização do exame preventivo do câncer cérvico-uterino em Porto Velho, estado de Rondônia, Brasil. Rev Pan-Amaz Saúde. 2011; 2(2):45-50.

2. Aguilar RP, Soares DA. Barreiras à realização do exame Papanicolau: perspectivas de usuárias e profissionais da estratégia de Saúde da Família da cidade de Vitória da Conquista-BA. Physis revista de Saúde Coletiva. 2015; 25(2):359-379.

3. Brito-Silva K, Bezerra AFB, Chaves LDP, Tanaka OY. Integralidade no cuidado ao câncer do colo do útero: avaliação do acesso. Rev Saúde Pública 2014; 48(2):240248.

4. Brasil. Ministério da Saúde. Instituto Nacional de Câncer. Estimativa 2016: incidência de câncer no Brasil. Rio de Janeiro: INCA; 2015.

5. Brasil. Ministério da saúde. Secretaria de vigilância em saúde. Coordenação geral de informações e análises epidemiológicas. Sistema de informação sobre mortalidade. Informações de saúde. 2012.

6. Casarin MR, Piccoli JCE. Educação em saúde para prevenção do cãncer de colo do útero em muheres do município de Santo Ângelo/RS. Cien. Saude Colet. 2011; 16(9):3925-3932.

7. Malta DC, Jorge AO. Análise de tendência de citologia oncótica e mamografia das capitais brasileiras. Rev Cien Cult. 2014; 66(1):25-29.

8. Rico AM, Iriart JAB. "Tem mulher, tem preventivo": sentidos das práticas preventivas do câncer do colo do útero entre mulheres de Salvador, Bahia, Brasil. Cad Saúde Pública. 2013; 29(9):1763-1773.

9. Moura EC, Morais Neto OL, Malta DC, Moura L, Silva $\mathrm{NN}$, Bernal R, et al. Vigilância de fatores de risco para doenças crônicas por inquérito telefônico nas capitais dos 26 estados brasileiros e no Distrito Federal (2006). Rev Bras Epidemiol. 2008; 11:20-37.

10. Graham K. Compensating for missing survey data. Michigan: Ann Arbor; 1983.

11. Brasil. Ministério da Saúde. Secretaria de Vigilância em Saúde. Departamento de Vigilância de Doenças e Agravos não Transmissíveis e Promoção da Saúde. Vigitel Brasil 2015: vigilância de fatores de risco e proteção para doenças crônicas por inquérito telefônico. Brasília: Ministério da Saúde; 2016.

12. Ponderação pelo método rake. Avaiable from: http:// portalarquivos.saude.gov.br/images/pdf/2014/ setembro/29/Nota-----pondera-----o-Rake.pdf. Accessed in 2017 (Jul 09).

13. World Health Organization. National Cancer Control Programmes: policies and managerial guidelines. $2 \mathrm{ed}$. Geneva: WHO; 2002.

14. Vale DBAP, Morais SS, Pimenta AL, Zeferino LC. Avaliação do rastreamento do câncer do colo do útero na Estratégia Saúde da Família no Município de Amparo, São Paulo, Brasil. Cad Saude Publica. 2010; 26(2):383-390. 
15. Cesar JA, Horta BL, Gomes G, Houlthausen RS, Willrich RM, Kaercher A, et al. Fatores associados a não realização do exame citopatológico de colo uterino no extremo Sul do Brasil. Cad Saude Publica. 2003;19(5):1365-1372.

16. Silva DSM, Silva AMN, Brito LMO, Gomes SRL, Nascimento MDSB, Chein MBC. Rastreamento do câncer do colo do útero no Estado do Maranhão, Brasil. Cienc Saúde Coletiva. 2014; 19(4):1163-1170.

17. Thuler LCS, Bergmann A, Casado L. Perfil dos pacientes com câncer do colo do útero no Brasil, 2000-2009: estudo de base secundária. Rev Brasileira de Cancerologia. 2012; 58(3):351-357.

18. Amorim VMSL, Barros MBA, César CLG, Carandina L, Goldbaum M. Fatores associados a não realização do exame de papanicolaou: um estudo de base populacional no Município de Campinas, São Paulo, Brasil. Cad Saude Publica. 2006; 22(11):2329-2338.
19. Monteiro CA, Moura EC, Jaime PC, Claro RM. Validade de indicadores do consumo de alimentos e bebidas obtidos por inquérito telefônico. Rev Saude Publica 2008 ago; 42(4):582-589.

20. Francisco PMSB, Barros MBA, Segri NJ, Alves MCGP. Comparação de estimativas de inquéritos de base populacional. Rev Saúde Pública 2013; 47(1):60-68.

\section{CORRESPONDÊNCIA}

Ana Paula Leite Moreira

Rua Aristides Villar, 182, Funcionários I

João Pessoa - PB. CEP.: 58087-090.

Email: anapaulanutrifisio@hotmail.com 